\title{
EYE BLINK CONTROL OF APPLIANCES AND HEART MONITOR FOR PARALYTIC AND ELDERLY
}

\author{
Dr. H S Prasantha \\ Professor, Department of E\&CE, Nitte Meenakshi Institute of Technology Bangalore, \\ Affiliated to VTU Belgaum, Karnataka
}

Meghana Prakash, Nidhishree Hegde, Niveda Giridharan, Rakshitha K P

Undergraduate Student, Department of E\&CE, Nitte Meenakshi Institute of Technology Bangalore

Affiliated to VTU Belgaum, Karnataka

\begin{abstract}
Mobility is defined as the ability to move freely and easily. The ability to move independently is vital for all life functions and plays a major role in maintaining mental health. Paralysis is a loss of muscle function in a part or full body of the patient. In every patient suffering from immobility, eye is commonly found to be the only organ in working condition. Hence, the proposed paper presents an automation system to detect and count the number of blinks of the patient using image processing to detect blinks to trigger different appliances of the house along with a health monitor that alerts the caretaker or neighbors in times of emergencies. Blink detection is a concept that has undergone multiple algorithms and approaches, the previous ones made use of artificial neural network (template matching) which involved subjective training of every patient's eyes. Image processing approach sets the proposed paper apart from the rest. Although this paper aimed at developing an automation system for the aid of paralysis patients, practically it can be used for the aid of any patient suffering from movement disability.
\end{abstract}

Keywords - Eye blinks, Face detection, Paralysis patient, Health, control

\section{INTRODUCTION}

Paralysis is a medical condition in which a person loses control of a particular group of muscles in the body or sometimes, entire body. This condition arises when there is erroneous transmission and reception of signals between brain and muscles. Paralysis is of four types: Monoplegia (affects one arm or one leg), Hemiplegia (affects one leg and one arm on the same side of the body), Paraplegia (affects both legs) and Quadriplegia (affects both legs and both arms). Hence it can be said that in all types of paralysis, one of the legs is affected. Image processing is the use of computer algorithm to get an enhanced image or to extract some useful information from it. Nowadays, image processing is among rapidly growing technologies. Blink detection is a concept that has undergone multiple algorithms and approaches, the previous ones made use of artificial neural network (template matching) which involved subjective training of every patient's eyes. The proposed paper intends on counting the number of blinks of a patient incapable of movement (paralysis, senior citizens, etc.) To accomplish this task, a camera will be set up in front of the patient's face which will capture his face at all times and the system analyses the number of intentional blinks at once wherein each number of blinks are assigned to a controller that gives different outputs depending on the database. As it is important to monitor the general wellbeing of such individuals, the paper also intends to monitor the average heart rate of the person, using a biomedical signal processing algorithm.

There have been numerous attempts at eye movement recognition in automation aids for paralytic patients. Various researchers across the globe contributed towards control of appliances using eye blink detection. The efforts are made to present few contribution made by different authors. The algorithm provided by Ganjwani and Chhabria uses coherence algorithm to track eye movements and actuate wheelchair[1], Nagpure made use of a five stage algorithm for pupil tracking[3], Soukupov`A and Jan made use of Eye Aspect Ratio which is calculated area of an open eye to that of the closed eye to detect blinks[4]. George and Routray developed a fast and accurate algorithm for eye localization and gaze tracking in low resolution images [5]. Sinha provide extensive information regarding circular Hough transform which was played a great role in the program[6]. Zheng and Yao worked on the algorithm for multi-angle face detection based on DP-Adaboost[7]. Lim, Shiue, Ho, Yu, Kao, Wang and Kuo researched on anxiety and depression in patients with traumatic spinal cord injury[9]. Lucena Oilveira Velsos and Pereira improved the face detection performance by applying skin detection in post processing[8]. Pan and Tomkins developed a real-time QRS detection algorithm[10]. T Rao, K Rao, Manikanta, and Kumar worked to distinguish between normal and abnormal ECG[11]. Porr developed a detection algorithm for the heart rate of ECG[12]. 
Nemati, Deen and Mondal developed a wearable wireless ECG sensor which can be used for long term applications[13]. Jindal, Saudagar, Ekta and Devi used Pan-Tompkin Algorithm to create a MATLAB based GUI for ECG arrhythmia detection [14]. Zangana worked face detection algorithm by combining three color model algorithms [15]. Udayashankar and others detected blinks using template matching and assigned different blinks to different appliances [2], but has drawbacks such as the detection efficiency falls when there is background movement and under low light conditions. The proposed paper intends on improving in such areas. Manjula BM and others contributed for artifact removal of Ballistocardiogram signal [16]

The rest of the paper is organized as follows. Proposed Methodology are explained in section II. Experimental results are presented in section III. Concluding remarks are given in section IV.

\section{PROPOSED ALGORITHM}

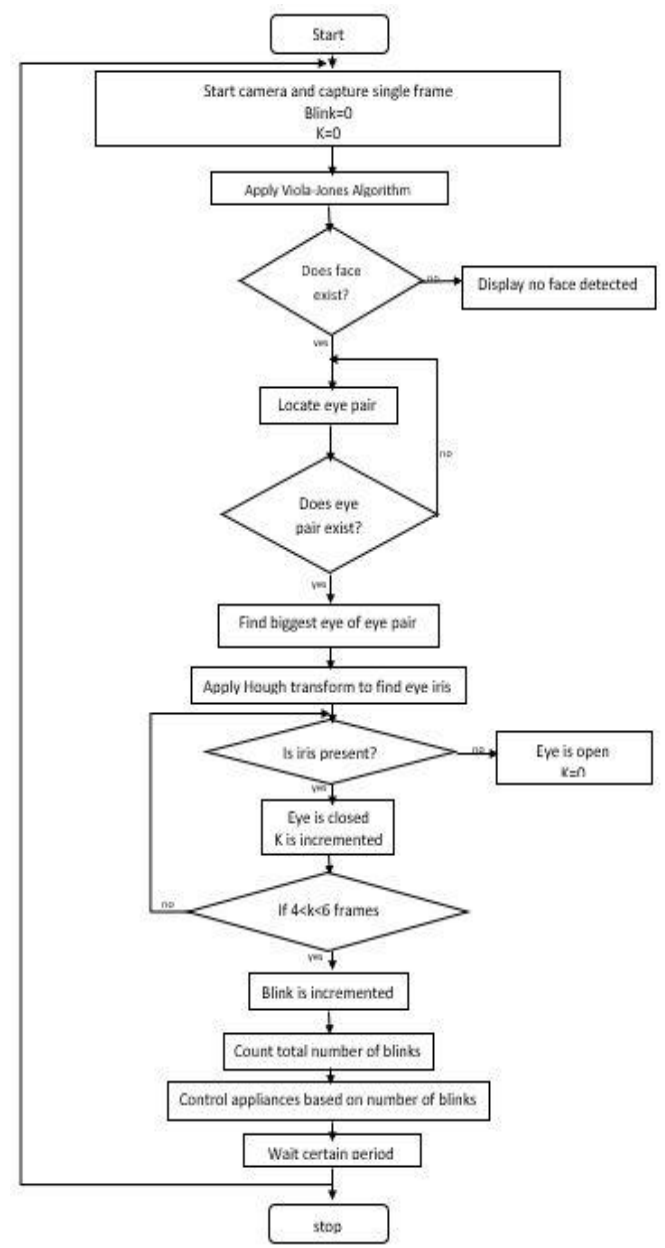

Figure 1: Flowchart of the blink detection

The figure 1 shows the flowchart of implementation of the proposed work. The first step is to switch on the camera and read the live video stream frame by frame. To every subsequent frame, Viola-Jones algorithm is applied to detect the face. If faces do exist, then the biggest face is chosen and then the algorithm locates the eye pair. The next step is to apply circular Hough transform to the segmented eye. If IRIS (circle) is present, it means the eye is open. If the eye is closed, the program keeps track of how long the eye remains closed with the help of variable $\mathrm{k}$ to decide if the blink is intentional or natural. If the eyes remain closed for approximately 1.8 seconds, then it is counted as an intentional blink. Finally, depending on the number of intentional blinks, each count is assigned to control a certain electrical appliance. It must be noted that, the health of the patient is being monitored alongside which will be explained in the upcoming sections.

\section{A. Face detection and Eye localization}

Face detection is a typical application of object detection where the face of an individual or a group of people is detected from an image or a video feed. Some of the methods used for face detection are Face detection using HoG features and SVM, Robust Face Detection Using the Hausdorff Distance, Detection of face using skin, and Viola-Jones algorithm (uses Haar Cascade feature detector.).

\section{B. Status of the eye}

In order to detect a blink, firstly the face is localized using Viola Jones detector and the eye region is segmented from the input webcam live feed, then knowing the status of the eye (open or closed) in each subsequent frame is required. The differentiating factor between an open and closed eye is the presence of a pupil in the frame. The pupil is in the shape of a circle in all human beings. If the eye is open in a particular frame, a circle (pupil) is seen and if the eye is closed, there will be no circle. Keeping this approach in mind, detection of circle in every frame of the eye became vital. To achieve this, circular Hough transform was used.

\section{Blink Count}

This program utilizes the unaffected eye to perform certain operations. Of all the actions that an eye performs, such as moving the iris in different directions or closing and opening the eyelids, blinking is the simplest action it performs. Hence, the number of blinks was chosen to be the criteria for control. An algorithm is modified to detect the face of the patient using Viola-Jones algorithm. When multiple faces are present, the face that is closest to the camera is considered. On detecting the face of the patient, the eye-pair is detected using the same algorithm. Of the eye-pair, the largest of the eyes is chosen for further operations. The average human blinks about 15 times a minute where each blink lasts about $1 / 10$ th of a second. This program differentiates between an intentional blink done by the patient and an involuntary blink that occurs naturally. The total number of intentional blinks are counted. A certain number of blinks is assigned to a basic appliance that the patient may want to control. In order to active the desired appliance, the patient need only to produce the required amount of intentional blinks. 


\section{Health Monitor}

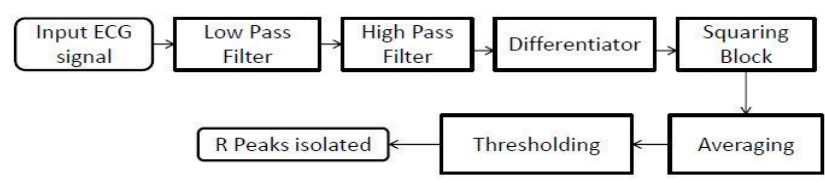

Figure 2: Block diagram of health monitor

The basic well-being of the subject is monitored by measuring the average heart rate. A standard ECG signal contains QRS complex, $\mathrm{P}$ and $\mathrm{T}$ waves, noise from electromyography signals, motion artifacts due to movement or contact with skin surface, in addition to other noise from equipments in the room or surroundings. In order to measure the heart rate, we need to extract the necessary signal, i.e. QRS complex, and more importantly R peaks.

We have used a modified version of the Pan and Tompkins's algorithm in order to do so. As the algorithm can only detect the QRS complex, but does not measure rate or speak of arrhythmia, it had to be modified to our requirements.

\section{Filtering}

To remove the noise signals, the ECG is first band-limited to a frequency range of $5-15 \mathrm{~Hz}$. The algorithm makes use of integer filters; hence a low pass filter and high pass filter are used in cascade. The signal is first passed through a second order low pass filter with cut off frequency set to $15 \mathrm{~Hz}$, to omit the electromyography signals as well as any power line interference.

To eliminate the low frequency noise due to $\mathrm{P}$ and $\mathrm{T}$ waves and baseline drift, a high pass filter is cascaded. The cut off frequency of the filter is $5 \mathrm{~Hz}$, and the delay to the all pass filter is $16 \mathrm{~T}$ (or $106.6 \mathrm{~ms}$, as the sampling rate of the filters used is $150 \mathrm{sps})$.

\section{Differentiator}

Once filtered, the signal is differentiated using a five point derivative filter to provide information on the slope of the QRS complexes. The signal produced is an alternation of spikes, with larger spikes being representative of the QRS complex. By doing so, we are able to enhance the QRS signal, while further attenuating the $\mathrm{P}$ and $\mathrm{T}$ waves.

\section{Squaring}

The differentiated signal is now squared to make all the data points positive and performs the non linear amplification of the output, emphasizing the frequency components due to QRS complex. Doing so strengthens the slope of the curve acquired by differentiation, and also limits the false positive peaks caused by $\mathrm{T}$ wave.

\section{Averaging}

Along with $\mathrm{R}$ peaks, additional wave features are also required in order to acquire the required outputs. A moving window integrator is applied to serve this purpose. The width of the window is chosen to be approximately the same size as the widest QRS complex. For a sample rate of 150 sps, a window width of 15 samples (corresponding to $100 \mathrm{~ms}$ ) is chosen.

\section{Thresholding and $\mathbf{R}$ peak Detection}

The R peaks in the ECG signal are detected after thresholding, which can be adjusted to adapt to changes in the morphology of the ECG and heart rate. Once these peaks are detected, R-R intervals can be computed, which are used to measure average heart rate as

Heart Rate $=60 * 1000 /$ [RR interval in ms]

Arrhythmia occurs when the heart rate of the individual falls below or exceeds the average resting rate of $60-100 \mathrm{bpm}$.

\section{E. Control of appliances}

A person affected with severe paralysis of any form is generally placed under 24/7 care and are catered to their every need. A basic need of a human inside their homes is illumination and circulation of air. A patient can control the lights and fan of the room they are present in by blinking a certain amount within the 100 frames time period. They can also turn on the fan to provide air circulation in the room. Along with appliances, an alarm can also be triggered by the patient to alert their caretaker.

The program can be executed over long periods of time. If the patient is to fall asleep while the program is executed, the algorithm detects that the eye has been closed for a long period of time and sets the blink count to zero. Once the patient wakes up, they need only to open their eyes and close it for approximately 2 seconds, to trigger an increase in the number of blinks. Even though these are very simple objects to control, it has 2 purposes: allow the user to gain some simple comfort by themselves and improve their mental health by giving them a sense of freedom and independence.

\section{IMPLEMENTATION DETAILS}

For the proposed work, the input image is captured by the webcam that is available in laptops. In order to interface the laptop webcam to MATLAB, MATLAB Support Package for USB Webcams was installed. This package allows MATLAB to access USB Video Class (UVC) webcam. This extends to built-in laptop camera or webcams that can be plugged into the computer via USB port. The resolution of the video feed is 640x360. The MATLAB R2017a version is used for the project. R2017a comes with a new product, Automated Driving System Toolbox, which helps design, simulate, and test ADAS and autonomous driving systems that is being extensively used in automotive industries. The input to the algorithm is the live video feed from the webcam captured at the rate of $30 \mathrm{fps}$. 


\section{EXPERIMENT AND RESULT}

The system was developed on Windows $10 \mathrm{PC}$ with $8^{\text {th }}$ gen Intel Core i5 processor and 4GB RAM. The entire coding and algorithm implementation was done on MATLAB. Although the input video feed is of $30 \mathrm{fps}$, for all the processing purposes it reduces to $4 \mathrm{fps}$. The system assigns 2 blinks to light, 3 blinks to fan, and so on which can be customized according the consumer requirements before installation. But 5 blinks is always set to actuate a loud buzzer to intimate the neighbors about the emergency and also sends an SOS mail to family/caretaker. Along with this, the system monitors the heart rate of the patient and on any occasion of irregularities, it immediately follows the emergency protocol by sending an SOS mail and actuating the buzzer. The entire program is linked to a simple MATLAB based GUI. This is to aid in the easy usage of the patient's family/caretaker.
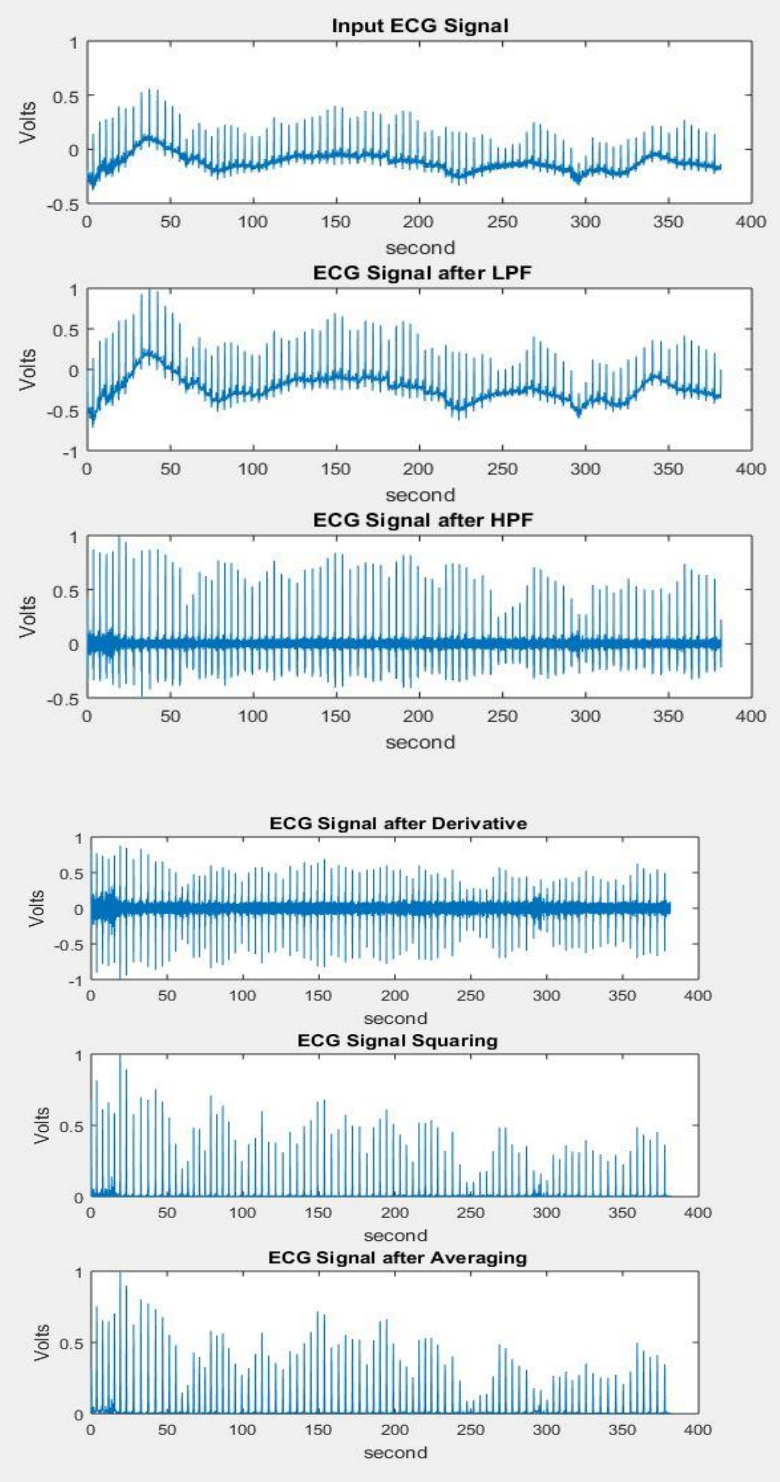
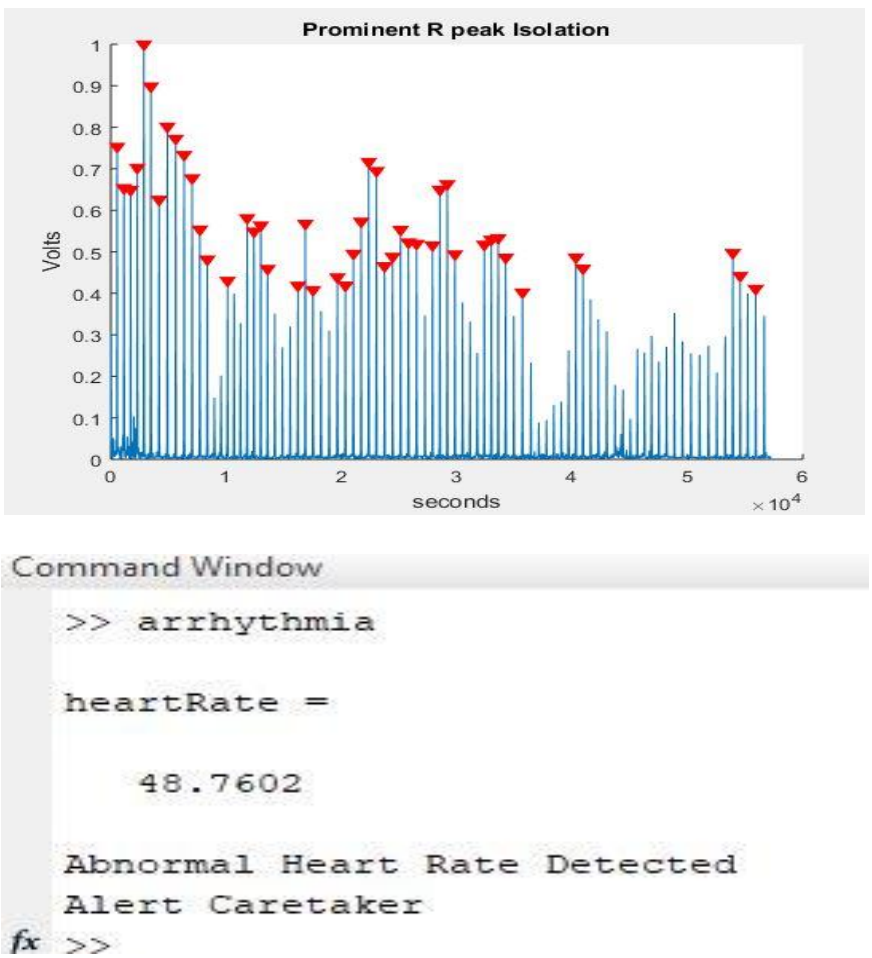

Figure 3: Abnormal heart rate

The irregularity in peaks and reduced heart rate observed implies that the heart is showing behaviors of arrhythmia. This poses a threat to the cardiac and over-all health of the individual. Hence the caretaker is alerted for immediate attention.

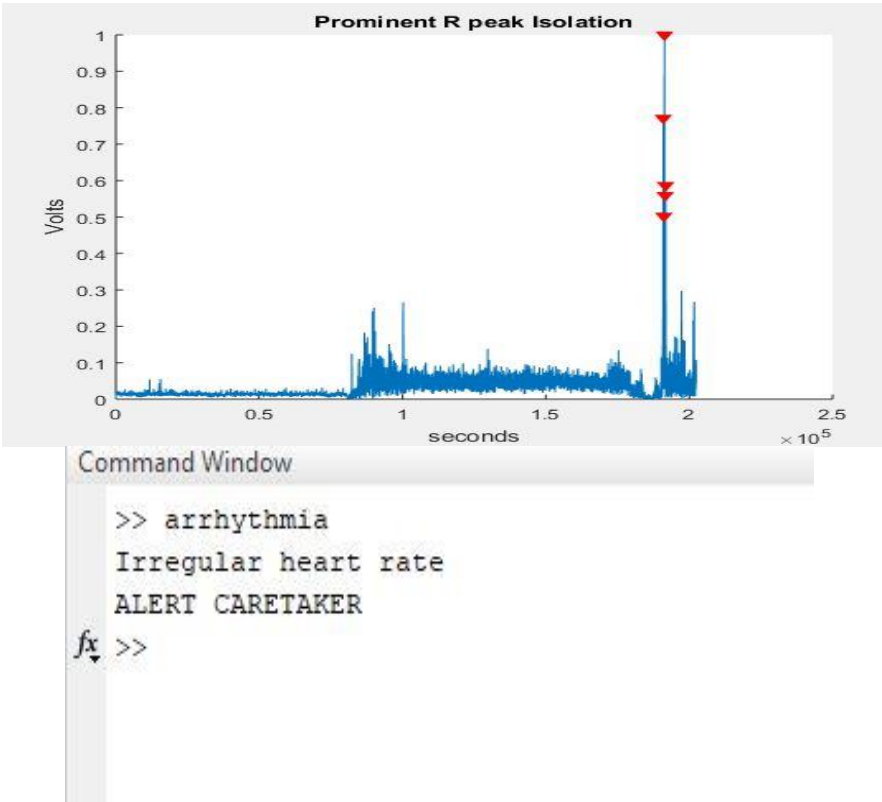

Figure 8: Erratic behavior

The observed signal shown in figures 7, 8 shows negligible peaks followed by a short-lived erratic signal. This could be a 
sign of arrhythmia or, it could mean that the sensors placed on the chest have been misplaced. In either of the cases this irregularity is a prompt to alert the caretaker for assistance.

\section{CONCLUSION}

While the system is aimed toward assisting patients affected with paralysis, it can be extended to fulfill the needs of other individuals as well. Although a small step, such a system allows the individual to regain some control over their own life. Furthermore, it allows the caretaker assigned to the individual to take short breaks during their day. The paper succeeded in developing a robust real time blink detection algorithm. There have been many algorithms developed for the purpose, some showing more accuracy than others. This paper presents a blink detection system using image processing. The paper also allows the monitoring of the patient's cardiac health using biomedical signal processing algorithms. Firstly, from the image of the individual, his/her face is localized and the right eye is segmented for use in the paper. Next phase involves the counting of intentional blinks and subsequent control of various home appliances, including an SOS message to alert the caretaker(s).Parallel to these processes, the health monitor, keeps a constant watch over the heart rate of the individual. If it were to fall under the standard range or show other signs of arrhythmia, an Alert message is immediately sent to the family or caretaker, irrespective of blink count.

\section{REFERENCES}

1. Poonam S Ganjwani and Sharda A Chhabria International Journal of Information Technology and Knowledge Management, "Eye motion tracking for wheelchair control," G H R C E, July-December 2012

2. Atish Udayashankar, Amit R Kowshik, Chandramouli S, H S Prasantha, "Assistance for the Paralyzed using Blink Detection", IEEE 2012 Fourth International Conference on Digital Home. DOI: 10.1109/ICDH.2012.9

3. Disha H Nagpure, Student, R.T.M.N.U, Shubhangi T Patil Lecturer, R.T.M.N.U, Snehal P Bujadkar Student of Computer Technology, R.T.M.N.U, Nagpur, India440022, "Eye Motion Detection Using Single Webcam for Person with Disabilities," International Journal of Engineering Research \& Technology (IJERT), March 2014

4. Tereza Soukupov'A And Jan `cech Center For Machine Perception, Department Of Cybernetics Faculty Of Electrical Engineering, Czech Technical University In Prague, "Real-Time Eye Blink Detection Using Facial Landmarks," 21st Computer Vision Winter Workshop, February 2016

5. Anjith George, Member, IEEE, and Aurobinda Routray, Member, IEEE, "Fast and Accurate Algorithm for Eye
Localization for Gaze Tracking in Low Resolution Images," 17 May 2016

6. Utkarsh Sinha, "Circular Hough Transform," 2010 [Online].Available: https://aishack.in/tutorials/circlehough-transform/

7. Ying-Ying Zheng, Jun Yao. School of Mechanical and Electronic Engineering and Automation, Shanghai University, Shanghai 200072, China. "Multi-angle Face detection based On DP-Adaboost", August 2015. DOI: 10.1007/s11633-014-0872-8

8. Oeslle Lucena, Italo de P. Oliveira, Luciana Veloso and Eanes Pereira. University of Campinas (UNICAMP), Federal University of Campina Grande (UFCG). "Improving face detection performance by skin detection post- processing”, October 2017. DOI: 10.1109/SIBGRAPI.2017.46

9. Sher-Wei Lim, Yow-Ling Shiue, Chung-Han Ho, ShouChun Yu, Pei-Hsin Kao, Jhi-Joung Wang, and Jinn-Rung Kuo, "Anxiety and Depression in Patients with Traumatic Spinal Cord Injury: A Nationwide Population-Based Cohort Study" 2017 Jan 12 [online] https://www.ncbi.nlm.nih.gov/pmc/articles/PMC5231351/

10. Jiapu Pan and Willis Tompkins, Senior Member, IEEE, “ $A$ real-time QRS detection algorithm”, IEEE Trans Biomed Eng. 1985 Mar.

11. Tirumala Rao, S. Koteswarao Rao, G. Manikanta and S. Ravi Kumar, Department of ECE, Vignan's Institute of Information Technology, Visakhapatnam - 530049, Andhra Pradesh, India. "Distinguishing Normal and Abnormal ECG”, Indian Journal of Science and Technology, Vol 9(10), DOI: 10.17485/ijst/2016/v9i10/85449, March 2016.

12. Bernd Porr, University of Glasgow,"Detecting the Heart Rate of ECG”, 2014 [Online], Available:https://biosignals.berndporr.me.uk/doku.php?id =octave:heartrate

13. Ebrahim Nemati, M. Jamal Deen, and Tapas Mondal, McMaster University, "A Wireless Wearable ECG Sensor for Long-Term Applications", IEEE Communications Magazine, 2012. DOI 0163-6804/12/\$25.00

14. Bhavna Jindal, Saudagar, Ekta, And Reeta Devi, "MATLAB Based GUI For ECG Arrhythmia Detection Using Pan-Tompkin Algorithm", 5th IEEE International Conference On Parallel, Distributed And Grid Computing (PDGC-2018), 20-22 Dec 2018, Solan, India. DOI 978-15386-6026-3/18/\$31

15. Hewa Majeed Zangana, "A new skin colour based face detection algorithm by combining three color model algorithms", IOSR Journal of Computer Engineering (IOSR-JCE) e-ISSN: 2278-0661, p-ISSN: 2278-8727, Volume 17, Issue 3, Ver. 1 (May - Jun. 2015), PP 06-12. DOI: $10.9790 / 0661-17310612$

16. Manjula B.M, Prashantha H.S , Goutham M.A, "Independent Component Analysis for Separation and Artifact Removal of Ballistocardiogram Signal", 
Published Online August 2020 in IJEAST (http://www.ijeast.com)

International Journal of Computer Sciences and Engineering (IJCSE), Vol.-7, Issue-7, July 2019

17. Dr. H S Prasantha, "NOVEL APPROACH FOR IMAGE COMPRESSION USING MODIFIED SVD”, International Journal of Creative Research Thoughts (IJCRT), Volume 8, Issue 8, Page 2234-2243, Aug 2020

18. Dr. H S Prasantha, "IMPLEMENTATION OF IMAGE COMPRESSION USING FAST COMPUTATION OF SVD ON DM642", International Journal of Creative Research Thoughts (IJCRT), Volume 8, Issue 8, Page 2364-2368, Aug 2020 